\title{
1 Nanopore sequencing of Giardia reveals widespread intra-isolate structural variation
}

2

3 Stephen M. J. Pollo ${ }^{1,2}$, Sarah J. Reiling ${ }^{3}$, Janneke Wit ${ }^{4}$, Matthew L. Workentine ${ }^{1}$, Rebecca A. Guy ${ }^{5}$, G.

4 William Batoff ${ }^{6}$, Janet Yee $^{6}$, Brent R. Dixon ${ }^{3}$, and James D. Wasmuth ${ }^{1,2^{*}}$

5

$6{ }^{1}$ Department of Ecosystem and Public Health, Faculty of Veterinary Medicine, University of Calgary,

7 Calgary, Alberta, Canada.

$8 \quad{ }^{2}$ Host-Parasite Interactions training program, University of Calgary, Calgary, Alberta, Canada.

$9 \quad{ }^{3}$ Bureau of Microbial Hazards, Food Directorate, Health Canada, Ottawa, Ontario, Canada.

$10{ }^{4}$ Department of Comparative Biology and Experimental Medicine, Faculty of Veterinary Medicine,

11 University of Calgary, Calgary, Alberta Canada.

$12{ }^{5}$ Division of Enteric Diseases, National Microbiology Laboratory, Public Health Agency of Canada,

13 Guelph, Ontario, Canada.

$14{ }^{6}$ Department of Biology, Biochemistry and Molecular Biology Program, Trent University, Peterborough,

15 Ontario, Canada.

$16 *$ Corresponding author

17

18 Email Addresses (in same order as authors):

19 stephen.pollo@ucalgary.ca, sarahdieerste@yahoo.de, jwit@ucalgary.ca,

20 matthew.workentine@ ucalgary.ca, rebecca.guy@canada.ca, gordonbatoff@trentu.ca, jyee@trentu.ca,

21 brent.dixon@canada.ca, jwasmuth@ucalgary.ca 
22 Key words: long read sequencing, MinION, structural variants, heterozygosity, parasite, polyploidy,

23 tetraploid, genome assembly

\section{Abstract}

25 Background: Genomes of the parasite Giardia duodenalis are relatively small for eukaryotic

26 genomes, yet there are only six publicly available. Difficulties in assembling the tetraploid $G$.

27 duodenalis genome from short read sequencing data likely contribute to this lack of genomic

28 information. We sequenced three isolates of G. duodenalis (AWB, BGS, and beaver) on the

29 Oxford Nanopore Technologies MinION whose long reads have the potential to address genomic

30 areas that are problematic for short reads.

31 Results: Using a hybrid approach that combines MinION long reads and Illumina short reads to

32 take advantage of the continuity of the long reads and the accuracy of the short reads we

33 generated reference quality genomes for each isolate. The genomes for two of the isolates were

34 evaluated against the available reference genomes for comparison. The third genome for which

35 there is no previous data was then assembled. The long reads were used to find structural

36 variants in each isolate to examine heterozygosity. Consistent with previous findings based on

37 SNPs, Giardia BGS was found to be considerably more heterozygous than the other isolates that

38 are from Assemblage A. We also find an enrichment of variant-specific surface proteins in some

39 of the structural variant regions.

40 Conclusions: Our results show that the MinION can be used to generate reference quality

41 genomes in Giardia and further be used to identify structural variant regions that are an

42 important source of genetic variation not previously examined in these parasites. 


\section{Background}

Giardia duodenalis (syn. Giardia lamblia or Giardia intestinalis) is a single-celled, eukaryotic, food and waterborne intestinal parasite that infects roughly 200 million people worldwide [1]. Infections can cause nausea, vomiting, diarrhea, and impaired growth and cognitive development [1]. The species $G$. duodenalis includes eight subtypes, named Assemblages A through $\mathrm{H}$, at least two of which are known to infect humans (A and B) [1]. The cells have two diploid nuclei each containing five chromosome pairs [2]. The haploid genome size is $~ 12.8 \mathrm{MB}$ [3]. Genome comparisons amongst assemblages of $G$. duodenalis found only $77 \%$ nucleotide and $78 \%$ amino acid identity in coding regions, suggesting the assemblages may represent different species [4]. Six isolates of G. duodenalis have reference genomes available [3].

Currently, whole genomes are sequenced using second generation technologies, third generation technologies, or strategies involving combinations of technologies (ex. combining PacBio and Illumina as in [5]). Second generation sequencing platforms produce high quality reads with low error rates $(0.1 \%$ for Illumina HiSeq) but short lengths (mean length $<250 \mathrm{bp}$ for Illumina HiSeq), which pose challenges for assembly programs resulting in more fragmented assemblies [6]. In contrast, third generation sequencing platforms produce much longer reads (mean length <10 000 bp for PacBio and MinION) but have higher error rates $(10-15 \%$ for PacBio and $>10 \%$ for MinION depending on the chemistry) [6-8]. These longer reads have the potential to resolve many genomic areas that are problematic for second generation data, such as repetitive and/or duplicated regions [8]. Importantly, eukaryotic genomes have many such repetitive and duplicated regions (as much as two thirds of the human genome may be repetitive elements [9]), making eukaryotic genomes especially good candidates for sequencing with third 
67 generation technologies. Moreover, third generation data is well suited for examining structural variants within a genome. In diploid and polyploid organisms the different copies of each chromosome can contain large scale differences, including insertions, deletions, duplications, 70 and translocations, in addition to variation at the single nucleotide level (SNPs). Collectively

71 called structural variants, they are a major source of genetic variation, thought to play a larger

72 role in phenotypic variation than SNPs, but are difficult to resolve using second generation data

73 [10-12]. The tetraploidy of Giardia trophozoites further complicates short read genome

74 assembly and structural variant detection methods because of the increased computational

75 complexity of constructing four haplotypes for each locus. For a review on the challenges associated with polyploid eukaryotic genomes see [13]. Our expectation is that long read

77 methods can detect and resolve the potentially three overlapping alternate alleles at any given 78 locus.

81 driven through small pores in a membrane by an electrical current which causes fluctuations in

82 the current in the pore [8]. Sensors measure these fluctuations, sending the data to a connected

83 computer for processing and storage [8]. Assembling genomes de novo from MinION data

84 involves basecalling of the squiggle files produced by the MinION during sequencing, assembly

85 of the long reads into draft genomes, and polishing of the assemblies.

87 Assemblage A isolate WB (hereafter referred to as Giardia AWB), G. duodenalis Assemblage B isolate GS (hereafter referred to as Giardia BGS), and G. duodenalis isolated from a beaver 
90 long and short reads, the long reads produced here were then used to investigate heterozygosity

91 in each isolate by detecting the structural variants in each genome.

\section{Data Description}

95 whole genome sequence data for three isolates of Giardia. In addition to assembling genomes for

96 the three isolates, we show the long read (MinION) data can be further used to detect structural

97 variant regions within each genome. The sequences can be accessed from the sequence read

98 archive (SRA) under accession number PRJNA561185.

\section{Analyses}

\section{Reference quality assemblies}

\section{Performance of ONT long reads}

104 with the exception of Run2, which was a second run conducted on a previously used flow cell

105 (Table 1). In addition to producing fewer reads, re-using the flow cell also resulted in lower

106 proportions of reads passing the quality threshold during basecalling with $64 \%$ and $81 \%$ of $1 \mathrm{D}$

107 reads passing in Run2 compared to 90 - 98\% of 1D reads passing in Runs 1, 3, and 4 (Table 1).

108 NanoOK [15] analysis of read error profiles showed that reads from Run2 have lower aligned

109 base identity and higher substitutions per 100 bases compared to the other runs (Table 2). 
111 the regions of reads that aligned to the reference genome (Table 2, aligned base identity) and a

$11224-46 \%$ error rate across the entirety of reads that aligned to the reference genome (Table 2 ,

113 overall base identity). The analysis also showed more deleted bases than inserted bases in the

114 reads (Table 2). Average and maximum read lengths for all runs are presented in Table 1.

115 Notably, the maximum 1D read length generated in the sequencing runs analyzed here was

$1161,132,445$ bases, though this read did not align to any Giardia reference genome nor did it have

117 significant BLAST hits longer than $\sim 45 \mathrm{bp}$ in the $\mathrm{nr}$ database (data not shown). It is presumably

118 a strand that got stuck but continued to generate (incorrect) sequence data.

Of the 39 long read de novo assemblies performed (13 input combinations x 3 assembly

121 sufficient numbers of reads to generate any contigs (AWB_2338_1D_smartdenovo,

122 AWB_2338_1Dsq for all three assemblers, and AWB_2331_2338_1D_smartdenovo). The

123 remaining assemblies were all polished with Nanopolish eight times and the evaluation metrics

124 were calculated for the nine resulting draft assemblies from each Giardia AWB and BGS

125 input/assembler combination for a total of 315 assemblies (Supplementary Table 1). The top

126 performing AWB and BGS assemblies for each metric are listed in Supplementary Table S2. No

127 assembly ranked first in more than two of the metrics. To further examine the effects of 1D vs

128 1Dsq input reads, pooling reads for the same isolate from multiple runs, assembly program, and

129 number of genome polishing iterations, for each metric the values for all the assemblies were

130 plotted (Supplementary Figs. S1 - S10). The average value and standard deviation for each

131 group were also calculated (Supplementary Tables S3-S10). Figure 1 shows the effects of 1D

132 vs 1Dsq input reads, assembly program, and number of genome polishing iterations on BGS 
assemblies for four of the metrics - the two that don't require a reference genome (number of

contigs and genome size), gene finding (BUSCO score), and accuracy measured as average

percent identity. The averages and standard deviations that correspond to Figure 1 can be found in Supplementary Tables S4, S8, and S10. The other metrics and the values for AWB assemblies show similar trends (Supplementary Figs. S1 - S10).

Table 2. Read error profiles for Giardia AWB and Giardia BGS MinION sequencing runs.

\begin{tabular}{|l|c|c|c|c|c|c|}
\hline Error Type & $\begin{array}{l}\text { AWB_01 } \\
\text { 50 Reads }\end{array}$ & $\begin{array}{c}\text { AWB_01 } \\
\text { 57 Reads }\end{array}$ & $\begin{array}{c}\text { AWB_23 } \\
\text { 31 Reads }\end{array}$ & $\begin{array}{c}\text { AWB_23 } \\
\text { 38 Reads }\end{array}$ & $\begin{array}{c}\text { BGS_22 } \\
\text { 37 Reads }\end{array}$ & $\begin{array}{c}\text { BGS_22 } \\
\text { 44 Reads }\end{array}$ \\
\hline $\begin{array}{l}\text { Proportion of Reads } \\
\text { Counted (\%) }\end{array}$ & 87.55 & 83.56 & 28.04 & 52.61 & 12.62 & 77.47 \\
\hline $\begin{array}{l}\text { Overall Base Identity } \\
(\%)\end{array}$ & 76.907 & 74.577 & 54.293 & 65.904 & 58.255 & 56.636 \\
\hline $\begin{array}{l}\text { Aligned Base } \\
\text { Identity (\%) }\end{array}$ & 90.526 & 89.352 & 83.076 & 83.915 & 91.429 & 89.954 \\
\hline $\begin{array}{l}\text { Identical Bases per } \\
\text { 100 }\end{array}$ & 80.430 & 78.338 & 71.024 & 71.597 & 80.855 & 78.834 \\
\hline $\begin{array}{l}\text { Inserted Bases per } \\
\text { 100 }\end{array}$ & 5.291 & 3.881 & 7.811 & 5.087 & 3.473 & 4.478 \\
\hline $\begin{array}{l}\text { Deleted Bases per } \\
\text { 100 }\end{array}$ & 5.860 & 8.450 & 6.758 & 9.592 & 8.105 & 7.886 \\
\hline Substitutions per 100 & 8.415 & 9.334 & 14.406 & 13.725 & 7.569 & 8.801 \\
\hline Mean Insertion & 1.638 & 1.462 & 1.755 & 1.480 & 1.482 & 1.530 \\
\hline Mean Deletion & 1.621 & 1.787 & 1.591 & 1.788 & 1.848 & 1.898 \\
\hline
\end{tabular}

Using NanoOK [15], 1D reads were aligned to the corresponding reference genome and the error

profiles of aligned reads were evaluated. NanoOK outputs read error profiles for each reference

contig. To get overall error profiles for all reads, the values for each contig were multiplied by

the proportion of total reads that aligned to that contig. The sum of these values for each error

metric were scaled according to the proportion of total sequencing reads that were used for

NanoOK's analysis. 
151 score (117, Supplementary Table S11) were constructed from a SMARTdenovo long read

152 assembly. For this reason, and because of the performance of the long read SMARTdenovo

153 assemblies in general (See Discussion of long read assemblies), the Giardia BGS and beaver

154 hybrid assemblies were constructed from Illumina reads and the SMARTdenovo assemblies of

155 the 1D MinION reads. The AWB hybrid assemblies outperformed their long read counterparts in

156 all metrics measured (Supplementary Tables S1 and S11) and, for all three isolates, the hybrid

157 assemblies had higher complete BUSCO scores than their corresponding long read assembly.

158 The best hybrid assembly for each isolate was selected for all further analysis on the basis of

159 maximum complete BUSCO score (AWB_hybrid_106_0150015723312338_1dsmartx0,

160 BGS_hybrid_gs3-20-2019_22372244_1dsmartx0, Beaver_hybrid_107218_2309_1dsmartx0).

161 For each of these assemblies, alignment to the AWB reference genome showed that the full

162 chromosome was recovered for chromosomes $1-4$ and the majority of chromosome 5 was also

163 recovered (Fig. 2).

We predicted structural variants from the long reads and hybrid assemblies to examine

167 the variation between the four copies of each chromosome in the Giardia isolates sequenced.

168 Giardia AWB, BGS, and beaver had 392, 1860, and 483 variants respectively (Table 3), which 
169

170

171

172

173

174

175

176

177

178

\begin{tabular}{|l|c|c|c|}
\hline & AWB & BGS & beaver \\
\hline Number of SVs & 392 & 1860 & 483 \\
\hline \# Duplications & $45(14520.4)$ & $185(48239.6)$ & $69(37535.0)$ \\
\hline \# Deletions & $46(15487.1)$ & $298(34454.6)$ & $74(46361.1)$ \\
\hline \# Inversions & $162(19437.9)$ & $746(28782.2)$ & $234(12866.7)$ \\
\hline $\begin{array}{l}\text { \# Inverted } \\
\text { Duplications }\end{array}$ & $2(2257.0)$ & $14(2680.1)$ & $0(0.0)$ \\
\hline \# Transversions & $104(2.3)$ & $436(20.8)$ & $46(4.0)$ \\
\hline \# Insertions & $33(299.6)$ & $181(596.4)$ & $60(286.9)$ \\
\hline $\begin{array}{l}\text { Proportion of } \\
\text { genome contained in } \\
\text { SVs }\end{array}$ & 0.1876 & 0.5662 & 0.3372 \\
\hline $\begin{array}{l}\text { Number of genes in } \\
\text { SVs }\end{array}$ & 2072 & 4151 & 3423 \\
\hline
\end{tabular}

affect 2072, 4151, and 3423 genes respectively. For each isolate, the full lists of predicted structural variants and genes affected by each variant can be found in Supplementary Tables S12 - S14. Notably among the genes affected are known virulence factors including variant-specific surface proteins (VSP), tenascins, and high cysteine membrane proteins [16]. In AWB, BGS, and beaver 39,97 , and 56 of the structural variants were found to have significantly more VSP than expected, respectively. Figure 3 shows alignments of the three hybrid genomes to the AWB reference genome with the predicted structural variants for each genome.

Table 3. Structural variants (SVs) in Giardia AWB, BGS, and beaver. Numbers in brackets are average lengths (bp) of the variants.
179

180

181

182

80 
The genome of Giardia beaver was assembled into 8 contigs totalling 11,467,485 bp. It

184

has a maximum contig length of $2.759 \mathrm{Mb}$ and an N50 of $1.965 \mathrm{Mb}$. One hundred thirteen complete BUSCOs were found out of 134 detected across the three Giardia isolates examined here. Giardia beaver has $49.56 \%$ GC content, similar to values found for Giardia AWB (49.0) and other assemblage $\mathrm{A}$ isolates $(49.25 ; 49.04)$ [2,17].

\section{Discussion}

\section{Long read assemblies and assemblers that lead to reference quality hybrid assemblies}

Among the three assemblers tested, the SMARTdenovo assemblies for both Giardia AWB and BGS showed the lowest variability in all metrics except average indel size (Fig. 1 and Supplementary Figs. S1 - S10). Moreover, the SMARTdenovo assemblies had the highest average values for average percent identity, BUSCO score, and proportion of reference covered 1X (where higher values indicate better performance) (Supplementary Table S1) and consistently strong performance in all metrics except average indel size (Fig. 1 and Supplementary Figs. S1 S10). Despite thirteen of the top performing assemblies (8 AWB, 5 BGS) being Abruijn assemblies (Supplementary Table S2), plotting values for each metric showed Abruijn had the most variable performance (Supplementary Figs. S1 - S10, Supplementary Tables S7 - S8). Canu assemblies generally performed somewhere between the SMARTdenovo and Abruijn assemblies (Supplementary Tables S7 - S8).

Analysis of the 207 AWB and 108 BGS assemblies indicates that the optimal long read only assembly pipeline for MinION sequenced Giardia is a SMARTdenovo assembly from 1D reads (either pooled or non-pooled input to reach sufficient genome coverage) followed by four 
or five rounds of polishing with Nanopolish (See Supplementary Material for discussion of 1D vs 1Dsq input reads, pooling different sequencing runs for the same organism, and number of rounds of genome polishing). However, it was the unpolished long read assemblies that resulted in the best hybrid assemblies (1D read, SMARTdenovo assembled, no polishing with Nanopolish; Supplementary Table S11). Interestingly, the BGS assemblies are larger than the reference BGS assembly that was generated from 454 data [4], potentially due to the fragmented nature of the reference assembly. The AWB and BGS hybrid assemblies generated here have higher complete BUSCO scores than the available reference genomes (117 for both hybrids vs 114 AWB reference and 116 BGS reference) and were assembled into very large pieces (AWB hybrid N50: 616 kb; BGS hybrid N50: 1,645 kb), suggesting they are of reference quality (Figs. 2 and 3). Moreover, the hybrid genome for Giardia beaver has a similarly high complete BUSCO score and similar contig numbers and contig lengths to the AWB and BGS hybrids, indicating that reference quality assemblies can be generated de novo for Giardia with as little as one ONT MinION and one multiplexed Illumina MiSeq sequencing run.

An optimal assembly pipeline for MinION data can change with each release of new programs specializing in handling long error prone reads. Already having the scripts to calculate the evaluation metrics used here makes re-evaluations easier to perform and enables evaluation of assembler performance that is current with each new program or version release. The typical publication process, from numerous drafts of a manuscript and peer-review, can be timeconsuming and not conducive to keeping such an analysis current. Therefore, a blog or community forum similar to an analysis on github of MinION basecalling programs [18] would be more appropriate. These media may also make it easier to discuss issues surrounding installation of these programs and running them in various computing environments. For 
example, some of the programs used here took up to a month to get installed and running properly. Having a current analysis of available long read assemblers would therefore also allow researchers to determine which programs are worth the time to get working and when it may be a better use of time to go with programs that need less configuration (like Canu which worked immediately) but will still perform adequately for the intended purpose.

\section{Structural variants reveal different levels of intra-isolate variation}

Despite having similar genome sizes, the three isolates examined here have very different total numbers of variants detected and proportions of their genomes that are within a structural variant region (Table 3, Fig. 3). When Giardia BGS was first sequenced, the authors noted a much higher allelic sequence heterozygosity than what was observed in AWB $(0.53 \%$ in BGS vs $0.01 \%$ in AWB) [4]. The same trend is observed in the structural variants here with BGS being considerably more heterozygous than AWB. The differences in allelic sequence heterozygosity were attributed to AWB and BGS being in different assemblages [4]. While the values for Giardia beaver (an assemblage A isolate) being more similar to AWB than BGS (Table 3) tentatively support the hypothesis that assemblage B is more heterozygous than assemblage A, many more genomes from each assemblage are needed to confirm it. Further, single cell sequencing could be used to examine the population structure of the isolates at a genetic level. Nonetheless, assemblage-specific variations in heterozygosity, or even isolate-specific variations in heterozygosity, will be important to consider in future comparisons between Giardia genomes. Previous genomic comparisons between assemblages [4] and within assemblages [19] have focused on SNPs and analyses of specific gene families. Including structural variant information 
250 provides a more complete picture of the heterozygosity and genetic diversity of each isolate by

251 capturing differences in gene dosage as well as gene content.

Recombination between different cells (outcrossing) within and between isolates of

255 Giardia has been suggested to occur through an as-yet undiscovered mechanism [20-23].

256 Outcrossing recombination events would allow for changes in gene copy number if the event

257 involved or encompassed a structural variant like a duplication or deletion. Alternatively, large

258 inversions can prevent recombination in the inverted areas [24], preventing gene flow during

259 recombination events in Giardia. These regions are therefore important to keep in mind in future

260 studies on recombination in Giardia as they may confound the analyses. Several dozen structural

261 variants from each of the isolates examined here were found to be significantly enriched for

262 VSP, supporting the suggestion that recombination is a potential source of VSP variation [25].

263 Expansions and contractions of this gene family through inheritance during outcrossing events of

264 duplicated or deleted loci that affect VSP could be an important factor in the number and

265 distribution of these genes between the various Giardia assemblages and isolates. As key surface

266 proteins involved in host immune evasion [26], these expansions and contractions of the VSP

267 repertoire could partially explain differences in pathogenicity between isolates. Moreover, as

268 mediators of the Giardia cell's interaction with its surrounding environment, expansions and

269 contractions of the VSP repertoire could affect host range. Alternatively, these genes could be

270 hotspots for recombination events that generate structural variants. Then in addition to their roles

271 as surface proteins they would also be potential factors influencing the evolution of Giardia

272 genomes. 


\section{Conclusions}

The present study demonstrates that high quality genomes can be generated for Giardia

276 for a few thousand dollars per genome, thus enabling future large scale comparative genomic

277 studies of the genus. Moreover, third generation long reads can be further used to investigate

278 heterozygosity and genome organization in Giardia despite its tetraploidy. We showed that

279 structural variant regions affect many genes notably virulence factors including VSP, suggesting

280 an important mechanism in the inheritance and distribution of these proteins among Giardia

281 isolates. Finally, we have generated a reference genome sequence for a new isolate, Giardia

282 beaver, with accompanying prediction of its structural variants.

\section{Methods}

Giardia duodenalis isolates

Giardia AWB (ATCC 30957) and Giardia BGS (ATCC 50580) were obtained from the

287 American Tissue Culture Collection, while Giardia beaver was a gift from Dr. Gaetan Faubert

288 from McGill University. Giardia trophozoites were grown in TYI-S-33 medium [27] in 16-mL

289 screw capped glass tubes incubated at $37^{\circ} \mathrm{C}$. 
294 tubes were chilled on ice for $5 \mathrm{~min}$ and the cells were collected by centrifugation at 1,100 x $\mathrm{g}$ for

$29515 \mathrm{~min}$ at $4^{\circ} \mathrm{C}$. Genomic DNA was extracted with DNAzol Reagent (ThermoFisher Scientific)

296 by following the manufacturer's instructions. Briefly, each cell pellet was resuspended and lysed

297 in DNAzol Reagent by gentle pipetting followed by a freeze $\left(30 \mathrm{~min}\right.$ at $\left.80^{\circ} \mathrm{C}\right)$ and thaw $(10 \mathrm{~min}$

298 at room temperature) step. The lysate was then centrifuged at $10,000 \mathrm{x} \mathrm{g}$ for $10 \mathrm{~min}$ at $4^{\circ} \mathrm{C}$ to

299 remove insoluble cell debris. The supernatant was transferred to a new tube and the DNA was

300 recovered by centrifugation of the supernatant at $4,000 \mathrm{xg}$ for $5 \mathrm{~min}$ at $4^{\circ} \mathrm{C}$. The DNA pellet was

301 washed twice with $75 \%$ ethanol then air-dried. The DNA was resuspended initially in $8 \mathrm{mM}$

$302 \mathrm{NaOH}$ then neutralized by addition of HEPES to a final concentration of $9 \mathrm{mM}$.

RNA was removed from the DNA sample by the addition of $1-2 \mu \mathrm{L}$ of $20 \mu \mathrm{g} / \mu \mathrm{L}$ RNase

the addition of ammonium acetate, incubation at $4^{\circ} \mathrm{C}$ for $20 \mathrm{~min}$, and centrifugation at $12,000 \mathrm{x} \mathrm{g}$

307 by the addition of $95 \%$ ethanol, incubation at room temperature for $5 \mathrm{~min}$, and centrifugation at 
316 carried out with live basecalling for $48 \mathrm{~h}$, or until no more strands were being sequenced. All

317 sequences were deposited in the sequence read archive (SRA) under accession number

PRJNA561185.

Illumina sequencing

Libraries were prepared using NexteraXT and paired-end sequenced on the MiSeq (v3,

2x300 cycles) or iSeq 100 (I1, 2x150 cycles) platforms according to manufacturer instructions

323 (Illumina Inc). All sequences were deposited in the SRA under accession number

324 PRJNA561185.

Long read basecalling, de novo assembly, and genome polishing reads. The flowcell and kit parameters were FLO-MIN107 and SQK-LSK308 respectively. The general command used to run Albacore was: full_ldsq_basecaller.py --flowcell 
1) all rounded to the nearest integer, and all other default settings (one polishing iteration, automatic detection of kmer size, minimum required overlap between reads of $5000 \mathrm{bp}$, automatic detection of minimum required kmer coverage, automatic detection of maximum 1), and setting gnuplotTested=true to bypass html output report construction. SMARTdenovo assemblies were conducted using default settings (kmer length for overlapping of 16 and

344 minimum required read length of 5000 bases). The general commands used to run each of the assemblers, with variable parameters written in upper case, were:

Abruijn: abruijn PATH/TO/READS out_nano COVERAGE_ESTIMATE --

Canu: canu -p UNIQUE_NAME genomeSize=12.8m -nanopore-raw

SMARTdenovo: smartdenovo.pl -p UNIQUE_NAME PATH/TO/READS >

UNIQUE_NAME.mak, followed by the command: make -f UNIQUE_NAME.mak

Genome polishing is an error correction step performed on assemblies generated from

third-generation data to compensate for the high error rate of the reads [8]. It involves re-

$$
\text { evaluating the base calls from the MinION squiggle files together with the read overlap }
$$

355 information from the assembly to improve base accuracy and correct small insertions and

356 deletions [32]. Here polishing was performed with the program Nanopolish (version 0.8.5)

357 following the directions for "computing a new consensus sequence for a draft assembly" [33].

358 Briefly, the draft genome was first indexed using BWA (version 0.7.15-r1140) [34] and the 
359 basecalled reads were aligned to the draft genome using BWA. SAMtools (version 1.6 using

360 htslib 1.6) [35] was then used to sort and index the alignment. Nanopolish then computed the

361 new consensus sequence in 50kb blocks in parallel, which were then merged into the polished

362 assembly. The general commands used to run Nanopolish were:

nanopolish index -d PATH/TO/FAST5/FILES PATH/TO/READS

bwa mem $-\mathrm{x}$ ont2d -t $8 \mathrm{PATH} / \mathrm{TO} / \mathrm{ASSEMBLY} / \mathrm{TO} / \mathrm{POLISH}$ PATH/TO/READS ।

samtools sort -o reads.sorted.bam -T reads.tmp

samtools index reads.sorted.bam

python /nanopolish/scripts/nanopolish_makerange.py

PATH/TO/ASSEMBLY/TO/POLISH | parallel --results

nanopolish.results -P 14 nanopolish variants --consensus

371 UNIQUE_NAME_polished_x $\{$ POLISHING_ITERATION $\} \cdot\{1\} . f a-w \quad\{1\}-r$

372 PATH/TO/READS -b reads.sorted.bam -g PATH/TO/ASSEMBLY/TO/POLISH

373 -t 4 --min-candidate-frequency 0.1

374 python /nanopolish/scripts/nanopolish_merge.py

375 UNIQUE_NAME_polished_x\$ $\{$ POLISHING_ITERATION $\} . *$ fa >

376 UNIQUE_NAME_polished_x\$ $\{$ POLISHING_ITERATION $\}$ genome.fa 
Read error profiles were examined for the six Giardia AWB and Giardia BGS runs using the program NanoOK (version v1.31) [15]. NanoOK extracts fasta sequences from the fast5 files produced by the MinION and aligns them to the reference genome using the LAST aligner (version 876) [36]. It then calculates error profiles for each set of reads that aligned to each metric the value for each contig was extracted from the .tex file produced by NanoOK and summed to yield the metric value with respect to all reads in the sequencing run. The sums were scaled according to the proportion of the total reads that were included in the metric calculation those that were mapped to the contigs - to yield the metric value for all reads used in the analysis.

The effects on final assembly quality were evaluated for the following parameters: 1D vs

392 1Dsq input reads, pooling reads for the same organism from multiple runs, assembly program, and number of genome polishing iterations. Firstly, 13 distinct input combinations, that represent

394 all permutations of pooling runs for the same organism for both 1D and 1Dsq reads, were used

395 for de novo assemblies: AWB_0157 1D reads, AWB_0157 1Dsq reads, AWB_0150_0157 1D

396 reads, AWB_0150_0157 1Dsq reads, AWB_2338 1D reads, AWB_2338 1Dsq reads,

AWB_2331_2338 1D reads, AWB_0150_0157_2331_2338 1D reads, AWB_0150_0157_2338

1Dsq reads, BGS_2244 1D reads, BGS_2244 1Dsq reads, BGS_2237_2244 1D reads, and

BGS_2237_2244 1Dsq reads (Table 1). Each of these input combinations was used to perform a

401 All of the resulting assemblies that produced contiguous sequences were polished with 
402 Nanopolish. Eight rounds of Nanopolish polishing were performed on the Canu and

403 SMARTdenovo assemblies and seven rounds were performed on the Abruijn assemblies (which

404 get polished once by Abruijn).

All assemblies and polished versions of the assemblies were aligned to the corresponding reference genome using the LAST aligner (version 876) [36] following the example for humanape alignments [37]. Briefly, the reference genome was indexed using LAST, then substitution and gap frequencies were determined using the last-train method [38]. Finally, alignments were performed using the lastal method and the determined substitution and gap frequencies. The resulting alignments were then filtered to retain only those alignments with an error probability <

$4111 \mathrm{e}^{-5}$. Giardia AWB assemblies were aligned to only the contigs from the reference genome 412 labelled GLCHR01, GLCHR02, GLCHR03, GLCHR04, and GLCHR05 (representing the five 413 chromosomes of Giardia duodenalis). Filtered alignments were converted to other file formats 414 (for metric calculation) using the maf-convert method in the LAST aligner.

Average percent identity was calculated from alignments in blasttab format by taking the sum of the percent identity multiplied by the alignment length for each aligned portion and

417 dividing that sum by the total alignment length. Proportion of mismatching bases was calculated

418 from alignments in psl format by taking the sum of mismatching bases for all aligned portions

419 divided by the total alignment length. Total number of indels per 1000 aligned bases was

420 calculated from alignments in psl format by taking the sum of the number of insertions in the

421 query and the number of insertions in the target for all aligned portions, dividing that sum by the

422 total alignment length and multiplying by 1000. Average size of indels was calculated from

423 alignments in psl format by taking the sum of the number of bases inserted in the query and the

424 number of bases inserted in the target for all aligned portions and dividing that sum by the total 
425 number of indels. The proportions of the reference covered $0,1,2,3$, or 4 times were calculated

426 using BEDtools (version v2.27.1) [39]. Alignments were first converted to SAM format and

427 SAMtools was used to sort the alignment and convert it to a bam file. The genomecov function

428 of BEDtools was then used to analyze the coverage of every base in the reference genome in the

429 alignment. The proportion of bases in the reference genome with $0,1,2,3$, and 4 fold coverage

430 in the assembly were retrieved.

432 for each assembly from the assembly fasta file. BUSCOs were calculated for each assembly

433 using BUSCO v3.0.2 (BLAST+ v2.6.0, HMMER v3.1b2, and AUGUSTUS v3.2.3), with the

434 eukaryote_odb9 dataset and default options (-sp fly) [40].

Average and standard deviation values for the groupings presented in the tables and

436 figures for each metric were calculated in $\mathrm{R}$ [41]. $\mathrm{R}$ was also used to construct the scatter plots

437 for the figures.

\section{Hybrid assemblies}

Hybrid genome assemblies were generated using the program Pilon (version 1.22) [42].

441 Briefly, short, highly accurate reads are mapped to a long-read assembly to correct for the higher

442 error rate in the long reads. For each hybrid assembly, the Illumina reads were mapped using

443 BWA to the long read assembly. After sorting and indexing the alignments with SAMtools, pilon

444 was run with default parameters to generate the hybrid assemblies. The general command to run

445 pilon was: 
pilon -Xmx200g --genome GENOME_TO_CORRECT --frags

447

BAM1.sorted.bam --frags BAM2.sorted.bam --output UNIQUE_NAME

The improvement of the hybrid assembly over the long read assembly from which it was

449 built was measured by the BUSCO scores of each (calculated as described above). BUSCO

450 scores were preferred because they do not depend on having a reference sequence and gene

451 finding depends on assembly accuracy. The best hybrid assembly for each isolate was deposited

452 at DDBJ/ENA/GenBank under the accession numbers VSRS00000000 (Giardia beaver),

453 VSRT00000000 (Giardia AWB), and VSRU00000000 (Giardia BGS). The versions described

454 in this paper are versions VSRS01000000, VSRT01000000, and VSRU01000000 respectively.

Structural variant prediction and analysis

458 Giardia isolate, the long reads were mapped to the best hybrid assembly using ngmlr v0.2.7. The

459 resulting alignments were sorted with SAMtools and the variants were called with sniffles

460 v1.0.10. The general commands to run ngmlr and sniffles were:

461

ngmlr -t 56 -r HYBRID_ASSEMBLY - - LONG_READS - o

462

UNIQUE_NAME_ngmlr.sam $-\mathrm{x}$ ont

sniffles -t 56 --genotype --cluster --report_seq -n -1 -m 
467 structural variants with the program exonerate v2.2.0 [43] and finding the genes overlapping the

468 variant regions using BEDtools. The general commands were:

469

exonerate -m protein2genome -q AWB_PROTEINS.gff -t

470

HYBRID_ASSEMBLY.fasta -M 250000 -n 1 --showalignment FALSE --

471 showvulgar FALSE --showtargetgff > UNIQUE_NAME.txt

472 sed '/^\#/d' UNIQUE_NAME.txt > UNIQUE_NAME.gff

473 sed ' $1,2 \mathrm{~d} ;$; d' UNIQUE NAME.gff > UNIQUE NAME 2.gff

474 bedtools intersect -a UNIQUE_NAME_SVs.vcf -b UNIQUE_NAME_2.gff -

475 wb > UNIQUE_NAME_intersect_vCf_genesonlynlgff.txt

For each variant type, the list of putatively affected genes was examined and genes of

477 interest were analyzed for enrichment in the variants. For each predicted variant, 10000 random

478 samples of the same size as the variant were selected from the genome. For each sample the

479 overlapping genes were found and the genes of interest were counted. The $95^{\text {th }}$ percentile was

480 calculated from the resulting distribution of genes of interest using the nearest-rank method to

481 find the count above which there is significant enrichment of the gene of interest (ie. the cutoff

482 for rejecting $\mathrm{H}_{0}$ ). The subsampling experiment was implemented in Java, the code for which is

483 available on github at https://github.com/StephenMJPollo/SV_Subsampling. 
487 SMARTdenovo (see discussion; commands are the same as in methods above). Illumina reads

488 were added to create a hybrid assembly as described above.

490 Availability of source code and requirements

491 Project name: SV_Subsampling

492 Project home page: https://github.com/StephenMJPollo/SV_Subsampling

493 Operating system: Linux

494 Programming Language: Java

495 Other requirements: BEDtools

497 Availability of supporting data and materials

499 hybrid assemblies generated are available from GenBank under the accession numbers

500 VSRS00000000 (Giardia beaver), VSRT00000000 (Giardia AWB), and VSRU00000000

501 (Giardia BGS). The versions described in this paper are versions VSRS01000000,

502 VSRT01000000, and VSRU01000000 respectively. All other supporting material will be 503 submitted to the GigaScience GigaDB database. 
506 Supplementary_Discussion: Additional discussion on long read only assemblies.

507 Supplementary Figures: Figures S1 - S10 with corresponding legends.

508 Supplementary Tables: Tables S1 - S15.

509

$510 \quad$ List of abbreviations

511 bp: base pairs; BUSCO: benchmarking universal single copy orthologs; ONT: Oxford Nanopore

512 Technologies; SNPs: single nucleotide polymorphisms; SRA: sequence read archive; SVs:

513 structural variants; VSP: variant-specific surface proteins.

515 Consent for publication

516 Not applicable.

518 Competing interests

519 The author(s) declare that they have no competing interests.

\section{Funding}

523 (OMAFRA \#FS2016-3010) to BRD, Alberta Agriculture and Forestry (AAF \#2016F013R) to

524 JDW, Natural Sciences and Engineering Research Council of Canada (NSERC) Discovery 
525 (\#222982) to JY, and a NSERC Visiting Fellowship in Canadian Government Laboratories to

526 SJR.

527

528 Authors' contributions

529 Resources and investigation: GWB, JW, and SJR. Investigation, formal analysis,

530 software, writing original draft, and visualization: SMJP. Funding acquisition and supervision:

531 RAG, JY, BRD, and JDW. Conceptualization and methodology: SMJP, SJR, MLW, RAG, BRD,

532 and JDW.

533

534 Acknowledgements

535 Not applicable

536

537 References

538 1. Certad G, Viscogliosi E, Chabé M, Cacciò SM. Pathogenic mechanisms of Cryptosporidium

539 and Giardia. Trends Parasitol. 2017;33:561-76.

540 2. Morrison HG, McArthur AG, Gillin FD, Aley SB, Adam RD, Olsen GJ, et al. Genomic

541 minimalism in the early diverging intestinal parasite Giardia lamblia. Science. 2007;317:1921-

5426.

543 3. Aurrecoechea C, Brestelli J, Brunk BP, Carlton JM, Dommer J, Fischer S, et al. GiardiaDB

544 and TrichDB: Integrated genomic resources for the eukaryotic protist pathogens Giardia lamblia 
and Trichomonas vaginalis. Nucleic Acids Res. 2009;37:526-30.

546 4. Franzén O, Jerlström-Hultqvist J, Castro E, Sherwood E, Ankarklev J, Reiner DS, et al. Draft

547 genome sequencing of Giardia intestinalis Assemblage B isolate GS: Is human giardiasis caused

548 by two different species? Plos Pathog. 2009;5:e1000560.

549 5. Stroehlein AJ, Korhonen PK, Chong TM, Lim YL, Chan KG, Webster B, et al. High-quality

550 Schistosoma haematobium genome achieved by single-molecule and long-range sequencing.

551 Gigascience. 2019;8:1-12.

552 6. Rhoads A, Au KF. PacBio sequencing and its applications. Genomics Proteomics

553 Bioinformatics. 2015;13:278-89.

554 7. Tyson JR, O’Neil NJ, Jain M, Olsen HE, Hieter P, Snutch TP. Whole genome sequencing and 555 assembly of a Caenorhabditis elegans genome with complex genomic rearrangements using the 556 MinION sequencing device. bioRxiv. 2017;

557 8. Lu H, Giordano F, Ning Z. Oxford nanopore minION sequencing and genome assembly.

558 Genomics Proteomics Bioinformatics. 2016;14:265-79.

559 9. de Koning APJ, Gu W, Castoe TA, Batzer MA, Pollock DD. Repetitive elements may

560 comprise over two-thirds of the human genome. PLoS Genet. 2011;7:e1002384.

561 10. Sedlazeck FJ, Rescheneder P, Smolka M, Fang H, Nattestad M, Von Haeseler A, et al.

562 Accurate detection of complex structural variations using single-molecule sequencing. Nat

563 Methods. 2018;15:461-8.

564 11. Jeffares DC, Jolly C, Hoti M, Speed D, Shaw L, Rallis C, et al. Transient structural variations 565 have strong effects on quantitative traits and reproductive isolation in fission yeast. Nat 
Commun. 2017;8:14061.

12. Weischenfeldt J, Symmons O, Spitz F, Korbel JO. Phenotypic impact of genomic structural variation: insights from and for human disease. Nat Rev Genet. 2013;14:125-38.

$570 \quad$ Plant Genome Sequence Assembly. Front Plant Sci. 2018;9:1-15.

571 14. Feng Y, Zhang Y, Ying C, Wang D, Du C. Nanopore-based fourth-generation DNA

572 sequencing technology. Genomics Proteomics Bioinformatics. 2015;13:4-16.

573 15. Leggett RM, Heavens D, Caccamo M, Clark MD, Davey RP. NanoOK: Multi-reference

574 alignment analysis of nanopore sequencing data, quality and error profiles. Bioinformatics.

$575 \quad 2016 ; 32: 142-4$.

576 16. Dubourg A, Xia D, Winpenny JP, Naimi S Al, Bouzid M, Sexton DW, et al. Giardia

577 secretome highlights secreted tenascins as a key component of pathogenesis. Gigascience.

$578 \quad 2018 ; 7: 1-13$.

579 17. Adam RD, Dahlstrom EW, Martens CA, Bruno DP, Barbian KD, Ricklefs SM, et al.

580 Genome Sequencing of Giardia lamblia Genotypes A2 and B Isolates (DH and GS) and

581 Comparative Analysis with the Genomes of Genotypes A1 and E (WB and Pig). Genome Biol

582 Evol. 2013;5:2498-511.

583 18. Wick R. A comparison of different Oxford Nanopore basecallers. 2017.

584 https://github.com/rrwick/Basecalling-comparison\#m.

585 19. Ankarklev J, Franzén O, Peirasmaki D, Jerlström-Hultqvist J, Lebbad M, Andersson J, et al.

586 Comparative genomic analyses of freshly isolated Giardia intestinalis assemblage A isolates. 
BMC Genomics. 2015;16:1-14.

20. Cooper MA, Sterling CR, Gilman RH, Cama V, Ortega Y, Adam RD. Molecular Analysis of

Household Transmission of Giardia lamblia in a Region of High Endemicity in Peru. J Infect

Dis. 2010;202:1713-21. resolution multilocus sequence typing of Giardia intestinalis Assemblage A isolates reveals

595 zoonotic transmission, clonal outbreaks and recombination. Infect Genet Evol. 2018;60:7-16.

596 23. Birky CW. Giardia Sex? Yes, but how and how much? Trends Parasitol. 2010. p. 70-4.

597 24. Wellenreuther M, Mérot C, Berdan E, Bernatchez L. Going beyond SNPs: The role of 598 structural genomic variants in adaptive evolution and species diversification. Mol Ecol. $599 \quad 2019 ; 28: 1203-9$.

602 Genomics. 2010;11:543-58.

603 26. Prucca CG, Slavin I, Quiroga R, Elías E V., Rivero FD, Saura A, et al. Antigenic variation in 604 Giardia lamblia is regulated by RNA interference. Nature. 2008;456:750-4.

605 27. Clark CG, Diamond LS. Methods for Cultivation of Luminal Parasitic Protists of Clinical 606 Importance. Clin mi. 2002;15:329-41.

607 28. Vera D. Dockerfile for the Albacore basecaller from Oxford Nanopore. 2017. 
608 https://github.com/dvera/albacore.

609 29. Lin Y, Yuan J, Kolmogorov M, Shen MW, Chaisson M, Pevzner PA. Assembly of long

610 error-prone reads using de Bruijn graphs. Proc Natl Acad Sci. 2016;113:E8396-405.

611 30. Koren S, Walenz BP, Berlin K, Miller JR, Bergman NH, Phillippy AM. Canu: scalable and

612 accurate long-read assembly via adaptive $k$-mer weighting and repeat separation. Genome Res.

$613 \quad 2017 ; 27: 722-36$.

614 31. Ruan J. Ultra-fast de novo assembler using long noisy reads. 2017.

615 https://github.com/ruanjue/smartdenovo.

616 32. Loman NJ, Quick J, Simpson JT. A complete bacterial genome assembled de novo using

617 only nanopore sequencing data. Nat Methods. 2015;12:733-6.

618 33. Simpson J. Signal-level algorithms for MinION data. 2017. https://github.com/jts/nanopolish.

619 34. Li H, Durbin R. Fast and accurate long-read alignment with Burrows-Wheeler transform.

620 Bioinformatics. 2010;26:589-95.

621 35. Cock PJA, Bonfield JK, Chevreux B, Li H. SAM/BAM format v1.5 extensions for de novo

622 assemblies. bioRxiv. 2015;00:1-3.

623 36. Kielbasa SM, Wan R, Sato K, Horton P, Frith MC. Adaptive seeds tame genomic sequence

624 comparison. Genome Res. 2011;21:487-93.

625 37. Mcfrith. last-genome-alignments. 2017. https://github.com/mcfrith/last-genome-alignments.

626 38. Hamada M, Ono Y, Asai K, Frith MC. Training alignment parameters for arbitrary

627 sequencers with LAST-TRAIN. Bioinformatics. 2017;33:926-8. 
628 39. Quinlan AR, Hall IM. BEDTools: A flexible suite of utilities for comparing genomic

629 features. Bioinformatics. 2010;26:841-2.

630 40. Simão FA, Waterhouse RM, Ioannidis P, Kriventseva E V, Zdobnov EM. BUSCO:

631 Assessing genome assembly and annotation completeness with single-copy orthologs.

632 Bioinformatics. 2015;31:3210-2.

633 41. R Core Team. R: A language and environment for statistical computing. 2013. http://www.r-

634 project.org/.

635 42. Walker BJ, Abeel T, Shea T, Priest M, Abouelliel A, Sakthikumar S, et al. Pilon: An

636 integrated tool for comprehensive microbial variant detection and genome assembly

637 improvement. PLoS One. 2014;9:e112963.

638 43. Slater GSC, Birney E. Automated generation of heuristics for biological sequence

639 comparison. BMC Bioinformatics. 2005;6:1-11.

640

641

642

643

644

645

646

647 
648 Figure 1. Performance metrics for all Giardia BGS long read assemblies. The title above each

649

650

651

652

653

654

655

656

657

658

659

660

661

662

663

664

665

666

667

668 scatterplot denotes the metric being plotted on the y-axis. The left column shows the differences between 1D (red Xs) vs 1Dsq (blue circles) data for each assembly protocol. Note that the data are paired. The middle column shows the assemblies separated by assembly program: abruijn (black Xs), canu (green circles), and SMARTdenovo (purple boxes). In the left and middle columns, the assemblies are randomly assigned along the $\mathrm{x}$-axis for visualization purposes, hence there are no units. The right column shows polished sets of assemblies with the $\mathrm{x}$-axis denoting how many times the draft assembly was polished. The dashed grey line shows the size of the Giardia BGS reference assembly.

Figure 2. Dotplots (Oxford Grids) of pairwise whole genome alignments between the Giardia AWB reference genome and A) the Giardia AWB hybrid genome, B) the Giardia beaver hybrid genome, and C) the Giardia BGS hybrid genome. Each of the five Giardia chromosomes from the reference genome is represented as a column and each contig from the hybrid genome is represented as a row. Contig names and dots in the plot coloured red represent forward alignments while contig names and dots coloured in blue are reverse alignments.

Figure 3. Whole genome alignments with predicted structural variants. The hybrid assembly contigs are shown as coloured boxes next to the reference Giardia AWB chromosome to which they align (black lines with vertical names beside each). Translucent purple boxes above the contigs show the locations and sizes of predicted structural variants in all three hybrid genomes. 
669 Note to reviewers: an interactive version of figure 3 that has filtering capabilities for viewing the

670 structural variants can be found at: http://pages.cpsc.ucalgary.ca/ stephen.pollo/Giardia_SV_Fig/

671 This version would be added to the GigaScience GigaDB database linked to the paper

672

673 Table 1 on next page should go between pages 5 and 6. 
Table 1. MinION sequencing run metadata, Albacore [28] basecalling results for both 1D and 1Dsq basecalling, and read statistics. "Pass" and "Fail" refer to reads that met or did not meet the quality threshold, respectively. Run 2 was conducted on a previously used flow cell after 64-72 $\mathrm{h}$ run time and so had few pores left.

\begin{tabular}{|c|c|c|c|c|c|c|c|c|}
\hline $\begin{array}{l}\text { Name Used in } \\
\text { this Document }\end{array}$ & AWB_0150 & AWB_0157 & AWB_2331 & AWB_2338 & Beaver_2302 & Beaver_2309 & BGS_2237 & BGS_2244 \\
\hline Run Name & SRRun1 & SRRun1 & SRRun2 & SRRun2 & SRRun3 & SRRun3 & SRRun4 & SRRun4 \\
\hline Run ID & $\begin{array}{l}\text { 20170720_01 } \\
\text { 50_GiardiaW } \\
\text { B_20170719 }\end{array}$ & $\begin{array}{l}\text { 20170720_01 } \\
\text { 57_GiardiaW } \\
\text { B_20170719 }\end{array}$ & $\begin{array}{l}\text { 20170721_23 } \\
\text { 31_GiardiaW } \\
\text { B_20170721 }\end{array}$ & $\begin{array}{l}\text { 20170721_23 } \\
\text { 38_GiardiaW } \\
\text { B_20170721 }\end{array}$ & $\begin{array}{c}\text { 20170726_23 } \\
\text { 02_GiardiaB } \\
\text { eaver_20170 } \\
726\end{array}$ & $\begin{array}{c}\text { 20170726_23 } \\
\text { 09_GiardiaB } \\
\text { eaver_20170 } \\
726\end{array}$ & $\begin{array}{l}\text { 20170731_22 } \\
\text { 37_GiardiaG } \\
\text { S_20170731 }\end{array}$ & $\begin{array}{l}\text { 20170731_22 } \\
\text { 44_GiardiaG } \\
\text { S_20170731 }\end{array}$ \\
\hline Isolate & $\begin{array}{l}\text { Giardia } \\
\text { AWB }\end{array}$ & $\begin{array}{l}\text { Giardia } \\
\text { AWB }\end{array}$ & $\begin{array}{c}\text { Giardia } \\
\text { AWB }\end{array}$ & $\begin{array}{c}\text { Giardia } \\
\text { AWB }\end{array}$ & $\begin{array}{c}\text { Giardia } \\
\text { beaver }\end{array}$ & $\begin{array}{c}\text { Giardia } \\
\text { beaver }\end{array}$ & Giardia BGS & Giardia BGS \\
\hline $\begin{array}{l}\text { Reference } \\
\text { Genome Size } \\
\text { (bp) }\end{array}$ & 12827416 & 12827416 & 12827416 & 12827416 & N/A & N/A & 11001532 & 11001532 \\
\hline $\begin{array}{l}\text { Total Number of } \\
\text { 1D Reads }\end{array}$ & 1225 & 329039 & 237 & 19531 & 1668 & 382740 & 1508 & 885046 \\
\hline $\begin{array}{l}\text { Number of 1D } \\
\text { Reads Pass }\end{array}$ & 1207 & 304219 & 152 & 15842 & 1603 & 354581 & 1449 & 804942 \\
\hline $\begin{array}{l}\text { Number of 1D } \\
\text { Reads Fail }\end{array}$ & 18 & 24820 & 85 & 3689 & 65 & 28159 & 59 & 80104 \\
\hline $\begin{array}{l}\text { Total Number of } \\
\text { IDsq Reads }\end{array}$ & 172 & 60156 & 16 & 1904 & 146 & 53553 & 212 & 143371 \\
\hline $\begin{array}{l}\text { Number of 1Dsq } \\
\text { Reads Pass }\end{array}$ & 68 & 25755 & 0 & 192 & 69 & 29349 & 124 & 62452 \\
\hline $\begin{array}{l}\text { Number of 1Dsq } \\
\text { Reads Fail }\end{array}$ & 104 & 34401 & 16 & 1712 & 77 & 24204 & 88 & 80919 \\
\hline $\begin{array}{l}\text { Average Length } \\
\text { of 1D Reads }\end{array}$ & 5066.15 & 7195.29 & 3450.08 & 6484.00 & 5113.00 & 8270.88 & 6534.03 & 9417.60 \\
\hline Longest 1D Read & 42781 & 470735 & 32138 & 330795 & 37229 & 1132445 & 56642 & 485807 \\
\hline $\begin{array}{l}\text { Average Length } \\
\text { of 1Dsq Reads }\end{array}$ & 5335.22 & 7685.61 & 2853.62 & 7344.74 & 5273.86 & 8472.84 & 5529.57 & 9829.82 \\
\hline $\begin{array}{l}\text { Longest 1Dsq } \\
\text { Read }\end{array}$ & 18489 & 43102 & 6523 & 32705 & 22740 & 59564 & 25876 & 66185 \\
\hline
\end{tabular}


Average Percent Identity

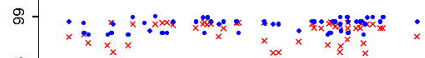

$\infty$

के

$\mathscr{G}-$\begin{tabular}{l}
$\times 10$ \\
$010 \mathrm{sa}$ \\
\hline
\end{tabular}

电

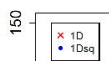

\&

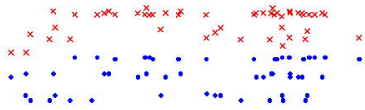

8

$\circ-$

$\stackrel{8}{\circ}-$

$\times \quad x^{x}$

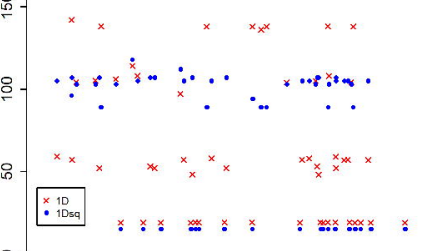

0

渵

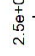

Genome Size

BUSCO score

Number of Contigs

\begin{tabular}{l}
$\times 10$ \\
$.10 \mathrm{sq}$ \\
\hline
\end{tabular}

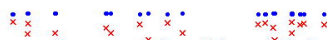

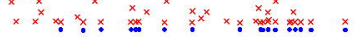

$\therefore . . .+\ldots$

Average Percent Identity

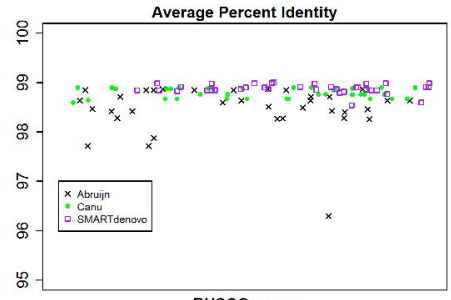

BUSCO score
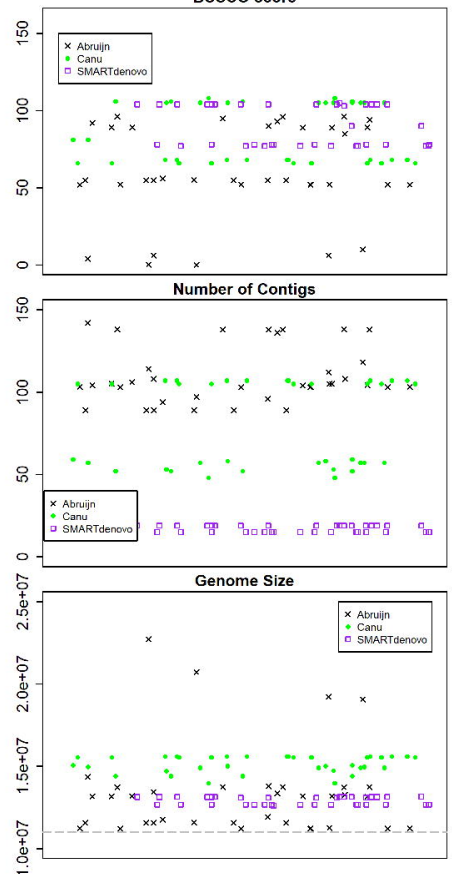

Average Percent Identity
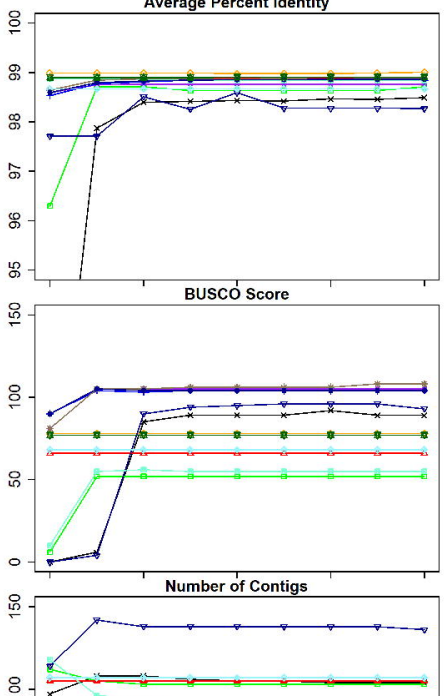

은

요
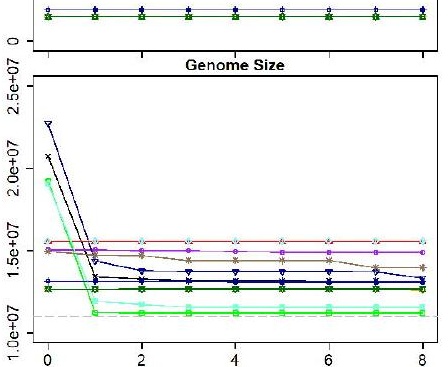


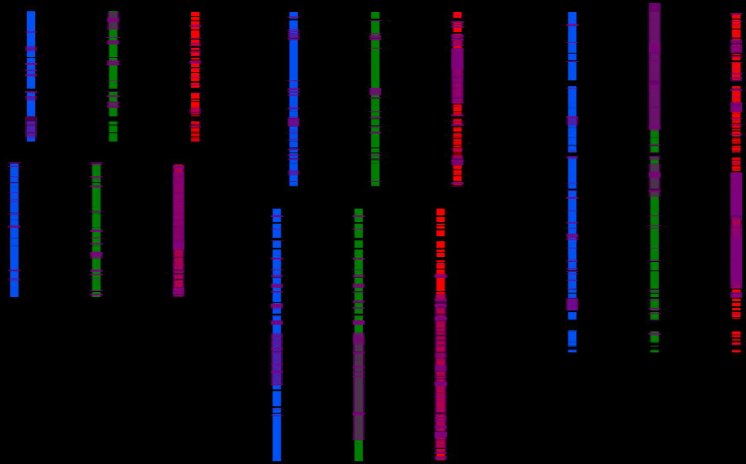

ㅁ

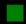

